\title{
Reef environments shape microbial partners in a highly connected coral population
}

\author{
Kriefall $\mathrm{NG}^{1}$, Kanke $\mathrm{MR}^{2}$, Aglyamova $\mathrm{GV}^{3}$, Davies $\mathrm{SW}^{1}$
}

$4 \quad{ }^{1}$ Biology Department, Boston University, Boston, MA, USA

$5{ }^{2}$ Department of Biomedical Sciences, College of Veterinary Medicine, Cornell University,

6 Ithaca, NY, USA

$7 \quad{ }^{3}$ Department of Integrative Biology, the University of Texas at Austin, Austin, TX, USA

\section{KEYWORDS}

10 Holobiont, reef zones, Acropora hyacinthus, Symbiodiniaceae, bacteria, French Polynesia

\section{ABSTRACT}

Corals from more thermally variable environments often fare better under thermal stress

14 compared to those from less thermally variable environments, an important finding given that

15 ocean warming threatens corals worldwide. Evidence is mounting that thermal tolerance can be

16 attributed to the coral itself, as well as microbial communities present within the holobiont (coral

17 host and its associated microorganisms). However, few studies have characterized how thermally

18 variable environments structure multiple holobiont members in situ. Here, using 2b-RAD

19 sequencing of the coral and metabarcoding of algal (ITS2) and bacterial (16S) communities, we

20 show evidence that reef zones (locales differing in proximity to shore, physical characteristics,

21 and environmental variability) structure algal and bacterial communities at different scales

22 within a highly connected coral population (Acropora hyacinthus) in French Polynesia. Fore reef

23 (more stable) algal communities were on average more diverse than the back reef (more 
24 variable), suggesting that variability constrains algal diversity. In contrast, microbial

25 communities were structured on smaller scales with site-specific indicator species and enriched

26 functions across reef zones. Our results illuminate how associations with unique microbial

27 communities can depend on spatial scale across highly dispersive coral populations, which may

28 have fitness consequences in thermally divergent regions and rapidly changing oceans.

30 INTRODUCTION

Anthropogenic climate change is causing widespread concerns across ecosystems.

32 Equatorial marine ectotherms, already living near their thermal limits, are especially vulnerable

33 (1). As a vivid example, tropical coral reefs are swiftly succumbing to the consequences of ocean

34 warming (2,3). Rising temperatures induce coral bleaching, a breakdown of the symbiosis

35 between corals and algae (Symbiodiniaceae) (2,3). If thermal stress is not relieved, bleaching and

36 subsequent lack of algal-derived nutrients can lead to coral death $(2,3)$. Widespread efforts are

37 underway to identify natural climate refugia as conservation and management targets (4).

38 Notably, areas of high frequency thermal variability are associated with coral bleaching

39 mitigation (2,3), however, the mechanisms underlying this phenomenon remain unclear.

The potential for adaptation and/or acclimatization to environmental variation spans

41 multiple members of the coral holobiont (unit encompassing coral hosts and their

42 microorganisms) $(5,6)$. Previous work has revealed genes associated with heat tolerance in corals

43 from contrasting thermal environments, and that this heat tolerance can be heritable $(7,8)$. In

44 addition, association with certain Symbiodiniaceae has been well-documented to confer

45 bleaching resistance $(9,10)$. Most recently, insights into the coral's microbiome have revealed

46 putative functions that mitigate the effects of thermal stress, such as nutrient cycling and 
47 immunity $(11,12)$. Indeed, inoculating Pocillopora damicornis with a consortium of potentially

48 beneficial bacteria prevented bleaching during both heat stress and pathogen challenge (13).

49 Clearly, to better understand coral resilience across environments, a holobiont perspective must

50 be taken.

51 Reef zones provide an effective natural system to study the coral holobiont under

52 divergent conditions, as strong environmental variation can be found even within $1 \mathrm{~km}(6,14)$.

53 Indeed, corals from more variable reef zones can exhibit increased thermal tolerance when

54 compared to corals from more stable reef zones $(2,15,16)$, but see (17). Significant genetic

55 differentiation across reef zones, as well as distinct algal and bacterial communities, has been

56 observed in brooding coral Pocillopora damicornis (6), highlighting the potential for multiple

57 holobiont members to be structured across short distances. However, a holistic view of how

58 multiple holobiont members vary across reef zones in a broadcast spawning coral, where genetic

59 differentiation across smaller scales is much less likely (18), remains less explored. In these

60 cases where life histories imply that corals will exhibit longer range migration, associations with

61 unique microbial communities might serve as a mechanism for corals to acclimatize to novel

62 environments.

63 Acropora hyacinthus is a dominant Indo-Pacific broadcast spawning coral that thrives

64 across reef zones (19). These zones differ in temperature, light, and nutrient levels, where the

65 back reef (close to shore) typically experiences greater variability when compared to the fore reef

66 (close to open ocean) (6,20). Here, we leverage reef zones in French Polynesia to ask how each

67 member of the A. hyacinthus holobiont is structured across fore reef and back reef environments.

68 Specifically we quantify: (1) coral host genetic structure and associated loci under selection, (2)

69 algal community composition and diversity, and (3) bacterial community composition, diversity, 
70 and functional profiles. Together, these data illuminate how these microbial members of the

71 coral holobiont vary across distinct environments and provide potential mechanisms by which

72 those from more variable environments may persist under climate change.

73

74 MATERIALS \& METHODS

75 Detailed materials and methods can be found in Supporting information.

76 Coral sampling and site characterization

77 In 2013, coral branch tips were collected from one fore reef $(\mathrm{F})$ and one back reef (B)

78 zone at each of three sites in French Polynesia: Tahiti (TNW), Mo'orea NW (MNW), and

79 Mo'orea SE (MSE) (Figure 1A; Table S1; CITES export permit \#FP1398700064-E). Samples

80 were preserved in $96 \%$ ethanol and maintained at $-20^{\circ} \mathrm{C}$ until processing. When possible, each

81 colony was photographed (no MSE-F photographs due to inclement weather) and surface area

82 was estimated using ImageJ (21) (e.g. Figure 1C). Colony surface area ( $\left.\mathrm{cm}^{2}\right)$ data were

83 transformed (Yeo-Johnson) and a one-way ANOVA tested for size differences between reef

84 zones.

85 Temperature data from two SBE 56 Temperature Sensors (Sea-Bird Scientific) labeled

86 'B1' (MNW-B) and 'P3' (MNW-F) sites were provided by Service d'Observation (SO) CORAIL

87 through CRIOBE. Hourly data from June $1^{\text {st }}, 2013$ through June $1^{\text {st }}, 2014$ were extracted. Mean

88 daily temperature and ranges were compared between reef zones using Mann-Whitney $U$ tests.

89 Host 2b-RAD genotyping

90 Holobiont tissue was isolated following (22). DNA concentrations were standardized to

$9125 \mathrm{ng} / \mu \mathrm{l}$ for 2b-RAD genotyping. 2b-RAD library preparations were modified from (23)

92 following https://github.com/z0on/2bRAD_denovo/. Briefly, DNA (100 ng) was digested with 
93 BcgI (New England Biolabs) and fragments were multiplexed using custom barcoded ligation

94 adapters. Samples were pooled and sequenced on three lanes of Illumina HiSeq 2500 (76

95 samples; Table S2) or one lane of Illumina HiSeq 4000 (60 samples; Table S2). Ten of 136

96 samples were technical replicates (Table S2). Raw and processed read count data are available in

97 Table S2.

All bioinformatic analyses are available at https://github.com/nicfall/moorea_holobiont.

99 Sequencing data were demultiplexed, de-duplicated when appropriate (see Table S2), and

100 filtered using FASTX-Toolkit (24). Filtered reads were mapped to the Acropora millepora

101 genome (25) using Bowtie2 (26). Analysis of Next Generation Sequencing Data (ANGSD) (27)

102 calculated genotype likelihoods using the following filters: only biallelic calls, only one best hit

103 per read, minimum mapping quality score of 20, minimum base quality score of 25 , maximum

104 strand bias $p$-value of $10^{-5}$, maximum heterozygosity bias $p$-value of $10^{-5}$, a minor allele

105 frequency of 0.1 , a minimum depth per site of 8 reads, and sites were required to be present in at 106 least $80 \%$ of samples. Pairwise identity-by-state (IBS) matrices were calculated and results

107 plotted as a distance dendrogram (Figure S1). Technical replicates and clones were removed,

108 leaving only one genotype representative. Samples with an average per-site depth of $<7$ reads

109 were removed to improve genotype likelihood confidence ( $n=10$ removed; Table S2). PCAngsd

110 (28) generated a covariance matrix for principal components analysis. Significance of

111 multivariate dispersion and location were assessed with vegan functions betadisper and adonis,

112 respectively (29). The ANGSD VCF file was converted into BED format using PLINK (30) and

113 run through ADMIXTURE (31) (K 1-5) where the lowest cross-validation error determined the

114 optimal ' $\mathrm{K}$ '. hierfstat (32) calculated global and pairwise $\mathrm{F}_{\mathrm{ST}}$ between all sites and reef zones. 
115 BayeScan (33) and OutFLANK (34) were used to identify single nucleotide polymorphism (SNP)

$116 \mathrm{~F}_{\mathrm{ST}}$ outliers.

117 Symbiodiniaceae metabarcoding

118 From a subset $(n=96 ; 16 /$ reef zone/site; Table S1) of samples used in host genotyping,

119 ITS2 metabarcoding was performed. ITS2 libraries were generated using a series of PCR

120 amplifications described in (35) and sequenced on Illumina Miseq (paired-end 250bp) at the

121 University of Texas at Austin. ITS2 data were pre-processed in bbmap (36) and cutadapt (37)

122 removed primer sequences. DADA2 (38) truncated reads, calculated error rates, de-duplicated

123 reads, inferred sequence variants, merged paired reads, and removed bimeras (38). One sample

124 failed quality filtering (Table S2). 118 non-bimeric amplicon sequence variants (ASVs) were

125 assigned taxonomy using the GeoSymbio database (39) with a minimum bootstrap confidence of

126 70. LULU curation algorithm (40) combined ASV counts with $99 \%$ sequence match. The

127 clustered ASV table was rarefied to 1,994 counts per sample, using vegan (29), which retained

$128 \sim 90 \%$ of samples (Table S2). Phyloseq (41) calculated three alpha diversity metrics: ASV

129 richness, Shannon index, and inverse Simpson's index. Mann-Whitney $U$ tests assessed

130 significance across reef zones overall and within each site. Linear regressions assessed

131 correlations between alpha diversity metrics and colony size.

132 MCMC.OTU (42) trimmed ASVs representing $<0.1 \%$ of reads or those present in only

133 one sample. A Principal Coordinate Analysis (PCoA) using Bray-Curtis dissimilarity was

134 conducted using phyloseq (41). Functions adonis and betadisper in vegan (29) evaluated sample

135 dissimilarity in multivariate space in terms of location and dispersion, respectively (99

136 permutations), contrasting reef zones and colony size within each site and pairwise.adonis (from

137 https://github.com/Jtrachsel/funfuns; 99 permutations) contrasted communities between sites. All 
138 analyses were repeated without rarefaction, using relative abundances to confirm results. In

139 relative abundance analyses, all but three samples were retained whose counts were at least 2.5

140 standard deviations lower than the mean (Table S2).

141 Bacterial metabarcoding

142 The same ITS2 sample subset was used to characterize the coral's microbiome using 16S

143 metabarcoding, except three samples which were substituted due to insufficient material and

144 three samples that failed to amplify ( $n=93,15-16 /$ site; Table S1, Table S2). The V4/V5 region

145 was amplified following (43). Pooled libraries were sequenced on an Illumina Miseq (paired-end

146250 bp) at North Carolina State University’s Genomic Sciences Library.

147 Pre-processing and dada2 analyses followed ITS2 protocols described above. 16S

148 taxonomy was assigned using the Silva v132 dataset (44). Using phyloseq (41), ASVs assigning

149 to family "Mitochondria", order "Chloroplast", or those failing to assign to kingdom "Bacteria"

150 were removed. Using vegan (29), the ASV table was rarefied to 12,000 reads, which retained

$151 \sim 90 \%$ of samples (9 samples removed; Table S2). Phyloseq (41) calculated three diversity

152 metrics: ASV richness, Shannon index, and inverse of Simpson's index. ASV richness and

153 inverse Simpson index were log-transformed and a one-way ANOVA with Tukey HSD tests

154 compared diversity metrics across sites and reef zones. MCMC.OTU (42) then trimmed ASVs

155 representing $<0.01 \%$ of counts or only present in one sample. Linear regressions assessed

156 correlations between alpha diversity metrics and colony size.

157 Sample dissimilarity was evaluated for the total bacterial community as described for

158 ITS2. These analyses were then repeated on $16 \mathrm{~S}$ data separated by microbiome (45) into core

159 (present in $>70 \%$ of samples) and accessory components, and finally repeated using unrarefied,

160 relative ASV abundances to confirm results. Indicspecies (46) (999 permutations) identified 
161 significant associations between ASVs and reef zones at each site, after multiple test correction

162 (47). Piphillin (48) predicted sample metagenomic content using the Kyoto Encyclopedia of

163 Genes and Genomes (KEGG) Database (49) and DESeq2 (50) identified differentially enriched

164 metagenomic content across reef zones within sites.

166 RESULTS

167 Detailed results can be found in Supporting information.

168 Site and coral colony characterization

169 Daily temperature range was higher in back reef relative to fore reef environments at

170 Mo'orea NW (Figure 1B). In contrast, mean daily temperature did not vary between reef zones.

171 Coral colony area was higher at the back reef than the fore reef at both Mo'orea NW and Tahiti

172 NW (Figure 1C).

173 Coral genetic structure and loci under selection

174 After quality filtering, reads per sample averaged 1.36 \pm 0.85 million (mean \pm SD) (Table

175 S2). Mean mapping efficiency across samples was 81.97 $\pm 4.03 \%$ (mean \pm SD) (Table S2). The

176 following samples were removed: 10 technical replicates (Figure S1), 2 incidental clones (Figure

$177 \mathrm{~S} 1$ ), and 10 samples having mean read depth <7/site (Table S2). 114 samples passed quality

178 filtering, which resulted in 3,594 SNPs. ADMIXTURE (31) determined that the optimal $\mathrm{K}=1$

179 (Figure S3). Principal component analysis confirmed this pattern, with sample overlap across

180 sites and reef zones (Figure S2). No differences in either multivariate location or dispersion

181 across sites, reef zones, or their interaction were observed (Figure S2). BayeScan (33) and

182 OutFLANK (34) found no $\mathrm{F}_{\mathrm{ST}}$ outliers. Pairwise $\mathrm{F}_{\mathrm{ST}}$ values between sites ranged between 0.011

183 and 0.018 (Table S3) and global $\mathrm{F}_{\mathrm{ST}}$ was 0.001. 
Average ITS2 counts were 4,681 $\pm 2,574$ (mean \pm SD) (Table S2). Most samples were

186 dominated by one of four ASVs assigned to ITS2 type Cladocopium C3k (mean relative

187 abundance $80.8 \%$ ). The second most abundant ITS2 type was Cladocopium Cspc (mean relative

188 abundance 12.5\%). Background symbiont types included two undescribed Cladocopium ASVs

189 and one Symbiodinium A1 ASV. Within all three sites, fore reef and back reef communities were

190 significantly different (Figure 2A). Data were not significantly dispersed in multivariate space,

191 with the exception of Tahiti NW, where back reef data were more dispersed than fore reef

192 (Figure 2A). Colony size significantly impacted community data at Tahiti NW only. With reef

193 zone data combined by site, algal communities were different between Mo'orea NW and Tahiti

194 NW, and between Mo'orea SE and Tahiti NW, but not between Mo'orea SE and Mo'orea NW

195 (Figure S4B).

196 Two metrics of alpha diversity indicated higher diversity in fore reef algal symbionts

197 when compared to back reef (Figure 2B). Within each site, there was higher diversity in fore reef

198 samples at Mo'orea NW and Mo'orea SE, but no differences observed between reef zones at

199 Tahiti NW (Figure 2C). There was only a significant difference in ASV richness across reef

200 zones at Mo'orea SE, with higher ASV richness in the fore reef. Colony size did not correlate

201 with any diversity metrics.

202 Bacterial community composition

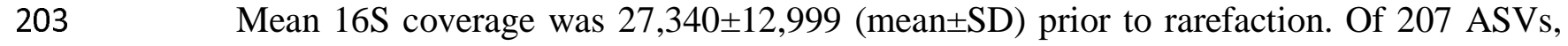

20410 comprised the core microbiome (Table S4). The most abundant member was an ASV

205 belonging to the Phylum Proteobacteria (35.76\%). The next two most abundant core ASVs 
207 (15\%). The remaining core and accessory ASVs averaged $<6 \%$.

211 between Mo'orea SE and Mo’orea NW and between Mo'orea SE and Tahiti NW, but not between Mo’orea NW and Tahiti NW (Figure S7).

Within each site, $16 \mathrm{~S}$ communities were significantly different between the fore reef and

214 back reef at Mo'orea SE and Tahiti NW, but not at Mo'orea NW (Figure 3A). There were no

215 significant differences in multivariate dispersion across reef zones or sites. Patterns did not

216 change when examining the core microbiome (Table S4). However, all reef zone comparisons at

217 all sites were significant when examining the accessory microbiome. There was no relationship

218 between colony size and community composition.

219 Indicator bacterial ASVs for each reef zone were largely unique to each site (Figure 3B).

220 Only one back reef indicator ASV was detected for Mo'orea NW (Pseudomonas sp.) and none

221 were detected at the fore reef. One back reef indicator ASV was significant for Mo'orea SE

222 (Family Simkaniaceae) and six for the fore reef (Phreatobacter sp., Lacibacter oligotrophus,

223 Rubrobacter sp., Curvibacter sp., Endozoicomonas sp., Order Obscuribacterales). There were

224 four back reef indicator ASVs for Tahiti NW (Phreatobacter sp., Lacibacter oligtrophus,

225 Enhydrobacter aerosaccus, Acidovorax), and one for the fore reef (Kingdom Bacteria).

226 Interestingly, two ASVs assigned to Phreatobacter and Lacibacter were back reef indicators at

227 Tahiti NW, but were fore reef indicators at Mo'orea SE (Figure 3B). 
230 functions were conserved and enriched in the back reef (vanillate monooxygenase ferredoxin

231 subunit; L,D-transpeptidase Erfk/SrfK; carboxynorspermidine decarboxylase; LysR family

232 transcriptional regulator, putative pyruvate carboxylase regulator), and one in the fore reef

233 (dipeptidase D); between Mo'orea NW and Tahiti NW, six functions were conserved and

234 enriched in the back reef (aldose sugar dehydrogenase; aminoglycoside 3-N-acetyltransferase I;

235 tetR/AcrR family transcriptional regulator, tetracycline repressor protein; streptomycin 3"-

236 adenylyltransferase; dihydropteroate synthase type 1; small multidrug resistance pump) (Figure

237 4; Table S5).

\section{DISCUSSION}

\section{Coral host gene flow is pervasive across reef zones and islands}

241 Studies where dispersive marine organisms display structured populations and outlier loci

242 across surprisingly small distances, likely due to environmental selection or dispersal barriers,

243 are accumulating rapidly $(6,7,15)$. Reef zones can play a notable role in this structuring.

244 Bongaerts et al. (14) and van Oppen et al. (6) found higher differentiation across habitats (reef

245 flat vs. slope; meters apart) than across sites (kilometers apart). In American Samoa, 114 outlier

246 loci were found in A. hyacinthus sampled from neighboring back reef pools, even though no

247 overall genetic structure was detected (8). Here, however, we observed that gene flow across

248 habitat types, sites, and even islands was pervasive (Figures S2, S3) and no outlier loci were

249 uncovered, suggesting that this A. hyacinthus population experiences high gene flow. This 
250 finding supports the growing consensus that the scale of gene flow is largely locale-specific and

251 not easily generalized, even within the same species $(51,52)$.

253 across greater oceanic distances (i.e. > $100 \mathrm{~km})$ than investigated here (25,53-55). Davies et al.

254 (18) demonstrated strong A. hyacinthus population connectivity across thousands of km, which is

255 consistent with our results between islands separated by only $\sim 15-30 \mathrm{~km}$. However, the

256 genotyping method used here, which re-sequences $\sim 1 \%$ of the genome (23), may have missed

257 putative loci under selection. Future work leveraging whole-genome resequencing or collections

258 from reef habitats with stronger environmental differences, e.g. (7), may uncover different

259 patterns. Nevertheless, our results indicate that molecular mechanisms beyond the host genome,

260 namely acclimatory changes such as changes in gene expression $(16,56,57)$ or changes in other

261 holobiont components $(5,58)$, are likely responsible for A. hyacinthus thriving across divergent

262 these reef zones.

263 More diverse Symbiodiniaceae communities in fore reef environments

264 Symbiodiniaceae communities were co-dominated by Cladocopium C3k and Cspc, with

265 background levels of other Cladocopium and Symbiodinium lineages (Figure S4A), consistent

266 with compositions found in Great Barrier Reef A. hyacinthus (59). Differences in relative

267 abundances of ITS2 types (Figures S4, S5) resulted in distinct community compositions across

268 reef zones (Figure 2A). These results are in line with numerous studies where ITS2 communities

269 are structured by reef habitats $(6,14,35,60)$. For instance, environmental variation across reefs in

270 Belize affected the types and abundances of symbionts hosted by Siderastrea siderea $(35,43)$.

271 Strikingly, van Oppen et al. (6) found that dominant ITS2 types within Pocillopora damicornis

272 were dependent on reef habitat, with C33 dominant on reef slopes and C42 on reef flats. Lastly, 
273 coral size is a proxy for age (61), and younger corals often have more diverse symbiont

274 communities than adults (62), however we found no consistent pattern between colony size and

275 ITS2 communities.

276 We observed higher alpha diversity in Symbiodiniaceae communities hosted by fore reef

277 corals for two of three sites (Figure 2B,C). Most ITS2 types were present in more samples and in

278 higher abundances in the fore reef (Figure S5). It is possible that selective pressures in the back

279 reef, e.g. increased thermal variability (Figure 1B), may constrain community evenness. As an

280 extreme example of reef environments constraining symbiont types, Bay and Palumbi (2014)

281 found that nearly all $A$. hyacinthus in a highly variable back reef pool were dominated by

282 Durisdinium while corals in the moderately variable pool hosted a mix of Cladocopium and

283 Durisdinium. In contrast, van Oppen et al. (2018) found higher symbiont alpha diversity in

284 Pocillopora damicornis living in more variable reef flats, highlighting that diversity patterns may

285 be site or species specific. In A. millepora, corals hosting more diverse symbiont communities

286 performed poorer under stress (63), but see (64), an area for follow-up investigations with

287 important implications for coral persistence.

288 Differences in A. hyacinthus Symbiodiniaceae communities may arise from

289 environmental structuring of free-living Symbiodinaceae sources or structuring by the host post-

290 uptake (65). Fine-scale environmental data collection at all sites as well as sampling of

291 Symbiodiniaceae sources would aid in disentangling the contributions of environmental factors

292 to community-level differences. In addition to temperature, nutrients, flow, and water quality

293 have all been suggested to influence Symbiodiniaceae communities across reef environments

$294(35,66,67)$. Differences in Symbiodiniaceae communities have important implications for the

295 coral, as different species of Symbiodiniaceae can impact host fitness $(9,10,68)$. It is also 
296 important to note that ITS2 is multicopy and it is difficult to interpret intra- and intergenomic

297 sequence diversity (69). Therefore, future work should employ more sensitive markers and

298 conduct population-level analyses of Cladocopium spp. to better understand algal symbiont

299 structuring across French Polynesia (9,70,71).

Bacterial taxa and functions are structured on smaller scales

$16 \mathrm{~S}$ total and core bacterial communities were distinct across reef zones at two of three sites (Figure 3A), while accessory bacterial communities were distinct across reef zones at all three sites. These results add to the growing body of literature demonstrating that local reef

304 environments are important drivers of coral microbiome structure $(6,43,72,73)$. Environments

305 differing in thermal variability have been shown to similarly structure bacterial communities, 306 ranging from A. hyacinthus in American Samoa (74) to Siderastrea spp in Belize (43). However, 307 other factors co-vary with temperature across reef zones (e.g. flow, water quality, light) and such

308 factors have also been implicated in coral microbiome community assembly (72,75). Potential 309 differences in environmental sources also exist across reefs as distinct bacterial water column 310 communities have been found across reef zones in Mo'orea (20). Further work will be required

311 to disentangle the structuring roles of biotic and abiotics factors for the coral holobiont. Indicator bacterial taxa for reef zones were specific to each of the three sites and some

313 taxa have been previously suggested to play roles in the coral holobiont (Figure 3B). For 314 instance, members of the genus Pseudomonas, an indicator taxon for the Mo'orea NW back reef

315 (Figure 3B), may be a beneficial microorganism in terms of sulfur cycling and antimicrobial 316 activity (12). The genus Acidovorax, an indicator taxon in Mo'orea SE back reef (Figure 3B), 317 also has nutrient cycling capabilities through nitrogen-related pathways (76). The genus 318 Curvibacter was an indicator in Mo'orea SE fore reef, and this taxon has been previously 
320 potentially due to uptake of host sugars with ABC-transporters $(79,80)$. Finally, genus

321 Endozoicomonas, which is found in marine invertebrates and vertebrates worldwide (81), was an

322 indicator taxon for Mo'orea SE fore reef. Within associations with Acroporid corals,

323 Endozoicomonas members have shown sensitivity to $\mathrm{pH}(82,83)$ and enrichment of KEGG

324 pathways associated with membrane and $\mathrm{ABC}$ transporters as well as cell signaling, motility, and

325 secretion (82). Overall, most identities and functional roles for coral-associated bacteria remain

326 unclear and represent a knowledge gap in microbial symbioses generally.

All three sites displayed differentially enriched bacterial functions across their reef zones

328 (Figure 4). Of the enriched functions consistent across reef zones at multiple sites (Figure 4B,C),

329 most shared between Mo'orea NW and Mo'orea SE were involved in metabolism, while half

330 shared between Mo'orea NW and Tahiti NW were involved in antimicrobial resistance (Table

331 S5). Both nutrient cycling and defense against pathogens have been postulated as mechanisms

332 that microbes use to help corals rapidly acclimate to their environment $(5,58)$. Two metabolic

333 functions identified here were related to amino acid metabolism (glutathione and

334 proline/arganine; Table S5) and these are consistent with findings in A. granulosa (84).

335 Interestingly, all but one of the functions conserved across multiple sites' reef zones were

336 enriched in more variable back reef sites. Our results corroborate Ziegler et al. (74), which

337 showed the majority of enriched bacterial functions within A. hyacinthus hosts between

338 moderately and highly variable reef pools were observed in the more variable environment,

339 perhaps due to environmental constraints. In addition, nutrient concentrations are typically

340 higher in the back reef around Mo'orea (20), perhaps necessitating the enrichment of functions

341 related to nutrient metabolism. Taken together, specific nutrient exchange and antimicrobial 
342 defense represent likely candidates for bacterial functions underlying coral success in the more

343 variable environment; however, much work remains to empirically link bacterial function to host

344 performance (74).

345 Conclusion

346 Understanding the scale of environmental selection for multiple members of the coral

347 holobiont remains critically important as intra-reef variability can determine how corals respond

348 to environmental stressors $(2,7)$. Here, we found evidence of environmentally structured

349 microorganism communities in a highly dispersive coral host. While we did not explore the

350 fitness consequences of hosting these divergent communities, evidence is accumulating that

351 microorganisms can help or hinder the coral host acclimate to rapid environmental change

352 (10,58). This work contributes to this burgeoning area of investigation by providing candidate

353 microbial taxa and functions that may facilitate or impede A. hyacinthus acclimatization to

354 divergent conditions. As oceans continue to warm, understanding the distributions and roles of

355 key microbial partners in holobiont resilience may hold the key to effective reef restoration.

\section{ACKNOWLEDGEMENTS}

358 We thank David Lecchini, Véronique Berteaux, and Elina Burns for assistance with fieldwork

359 and permit logistics; Rose Sulentic, Mark Lopez, Nana-Ama Anang, and Lara Laake-Emery for

360 wet lab assistance; the Methods in Ecological Genomic Analysis 2b-RAD class of 2014 for

361 assistance with library preparation; Mikhail Matz for contributions in experimental design,

362 molecular lab space, and bioinformatics; and finally Laura Tsang, Brianna Regan, and John

363 Weldon for help analyzing coral images. Funding was provided to SWD by the Institute des 
Récifs Coralliens du Pacifique with L’ecole Pratique des Hautes Etudes in addition to a start-up award from Boston University.

\section{DATA ACCESSIBILITY}

Raw 2b-RAD .fastq files are available at NCBI's SRA (PRJNAXXX). Raw ITS2 and 16S .fastq

files are available on NCBI's SRA (ITS2: PRJNA660421; 16S: PRJNA660779). Processed data

files and scripts used in analysis are available at https://github.com/Nicfall/moorea_holobiont.

\section{AUTHOR CONTRIBUTIONS}

374 SWD conceived of the study. SWD and MRK conducted all fieldwork. NGK, GVA, and SWD

375 conducted all wet lab work. NGK conducted all analyses with help from SWD and MRK. NGK

376 drafted the manuscript and all authors provided comments and approved the final version.

\section{REFERENCES}

379
1. Pinsky ML, Eikeset AM, McCauley DJ, Payne JL, Sunday JM. Greater vulnerability to warming of marine versus terrestrial ectotherms. Nature. 2019 May;569(7754):108-11.

2. Safaie A, Silbiger NJ, McClanahan TR, Pawlak G, Barshis DJ, Hench JL, et al. High frequency temperature variability reduces the risk of coral bleaching. Nature Communications [Internet]. 2018 Dec [cited 2019 May 20];9(1). Available from: http://www.nature.com/articles/s41467-018-04074-2

3. Sully S, Burkepile DE, Donovan MK, Hodgson G, van Woesik R. A global analysis of coral bleaching over the past two decades. Nature Communications. 2019 Mar 20;10(1):1264.

4. Cacciapaglia $\mathrm{C}$, Woesik $\mathrm{R}$ van. Reef-coral refugia in a rapidly changing ocean. Global Change Biology. 2015;21(6):2272-82.

5. Thompson JR, Rivera HE, Closek CJ, Medina M. Microbes in the coral holobiont: partners through evolution, development, and ecological interactions. Front Cell Infect Microbiol [Internet]. 2015 [cited 2019 May 23];4. Available from: https://www.frontiersin.org/articles/10.3389/fcimb.2014.00176/full 
6. van Oppen MJH, Bongaerts P, Frade P, Peplow LM, Boyd SE, Nim HT, et al. Adaptation to reef habitats through selection on the coral animal and its associated microbiome. Molecular Ecology. 2018 Jul;27(14):2956-71.

7. Bay RA, Palumbi SR. Multilocus Adaptation Associated with Heat Resistance in ReefBuilding Corals. Current Biology. 2014 Dec 15;24(24):2952-6.

8. Dixon GB, Davies SW, Aglyamova GV, Meyer E, Bay LK, Matz MV. Genomic determinants of coral heat tolerance across latitudes. Science. 2015 Jun 26;348(6242):1460-2.

9. Howells EJ, Beltran VH, Larsen NW, Bay LK, Willis BL, van Oppen MJH. Coral thermal tolerance shaped by local adaptation of photosymbionts. Nature Climate Change. 2012 Feb;2(2):116-20.

10. Berkelmans R, van Oppen MJH. The role of zooxanthellae in the thermal tolerance of corals: a 'nugget of hope' for coral reefs in an era of climate change. Proceedings of the Royal Society B: Biological Sciences. 2006 Sep 22;273(1599):2305-12.

11. Bourne DG, Morrow KM, Webster NS. Insights into the Coral Microbiome: Underpinning the Health and Resilience of Reef Ecosystems. Annual Review of Microbiology. 2016 Sep 8;70(1):317-40.

12. Peixoto RS, Rosado PM, Leite DC de A, Rosado AS, Bourne DG. Beneficial Microorganisms for Corals (BMC): Proposed Mechanisms for Coral Health and Resilience. Front Microbiol [Internet]. 2017 [cited 2020 Aug 10];8. Available from: https://www.frontiersin.org/articles/10.3389/fmicb.2017.00341/full

13. Rosado PM, Leite DCA, Duarte GAS, Chaloub RM, Jospin G, Nunes da Rocha U, et al. Marine probiotics: increasing coral resistance to bleaching through microbiome manipulation. The ISME Journal. 2019 Apr;13(4):921-36.

14. Bongaerts P, Riginos C, Ridgway T, Sampayo EM, van Oppen MJH, Englebert N, et al. Genetic Divergence across Habitats in the Widespread Coral Seriatopora hystrix and Its Associated Symbiodinium. Vollmer S, editor. PLoS ONE. 2010 May 27;5(5):e10871.

15. Barshis DJ, Birkeland C, Toonen RJ, Gates RD, Stillman JH. High-frequency temperature variability mirrors fixed differences in thermal limits of the massive coral Porites lobata. $\mathrm{J}$ Exp Biol. 2018 Dec 15;221(24):jeb188581.

16. Kenkel CD, Matz MV. Gene expression plasticity as a mechanism of coral adaptation to a variable environment. Nature Ecology \& Evolution. 2016 Nov 7;1(1):0014.

17. Klepac CN, Barshis DJ. Reduced thermal tolerance of massive coral species in a highly variable environment. Proc R Soc B. 2020 Aug 26;287(1933):20201379.

18. Davies SW, Treml EA, Kenkel CD, Matz MV. Exploring the role of Micronesian islands in the maintenance of coral genetic diversity in the Pacific Ocean. Molecular Ecology. 2015;24(1):70-82.

19. Linares C, Pratchett M, Coker D. Recolonisation of Acropora hyacinthus following climate-induced coral bleaching on the Great Barrier Reef. Marine Ecology Progress Series. 2011 Oct 5;438:97-104.

20. Leichter J, Alldredge A, Bernardi G, Brooks A, Carlson C, Carpenter R, et al. Biological and Physical Interactions on a Tropical Island Coral Reef: Transport and Retention Processes on Moorea, French Polynesia. oceanog. 2013 Sep 1;26(3):52-63.

21. Schneider CA, Rasband WS, Eliceiri KW. NIH Image to ImageJ: 25 years of image analysis. Nature Methods. 2012 Jul;9(7):671-5. 
22. Davies SW, Rahman M, Meyer E, Green EA, Buschiazzo E, Medina M, et al. Novel polymorphic microsatellite markers for population genetics of the endangered Caribbean star coral, Montastraea faveolata. Marine Biodiversity. 2013 Jun;43(2):167-72.

23. Wang S, Meyer E, McKay JK, Matz MV. 2b-RAD: a simple and flexible method for genome-wide genotyping. Nat Methods. 2012 Aug;9(8):808-10.

24. Hannon GJ. FASTX-Toolkit [Internet]. 2010. Available from: http://hannonlab.cshl.edu/fastx_toolkit

25. Fuller ZL, Mocellin VJL, Morris LA, Cantin N, Shepherd J, Sarre L, et al. Population genetics of the coral Acropora millepora: Toward genomic prediction of bleaching. $2020 ; 11$.

26. Langmead B, Salzberg SL. Fast gapped-read alignment with Bowtie 2. Nat Methods. 2012 Apr;9(4):357-9.

27. Korneliussen TS, Albrechtsen A, Nielsen R. ANGSD: Analysis of Next Generation Sequencing Data. BMC Bioinformatics. 2014 Nov 25;15(1):356.

28. Meisner J, Albrechtsen A. Inferring Population Structure and Admixture Proportions in Low-Depth NGS Data. Genetics. 2018 Oct 1;210(2):719-31.

29. Oksanen J, Guillaume Blanchet F, Friendly M, Kindt R, Legendre P, McGlinn D, et al. vegan: Community Ecology Package [Internet]. 2019. Available from: https:/CRAN.Rproject.org/package=vegan

30. Purcell S, Neale B, Todd-Brown K, Thomas L, Ferreira MAR, Bender D, et al. PLINK: A Tool Set for Whole-Genome Association and Population-Based Linkage Analyses. Am J Hum Genet. 2007 Sep;81(3):559-75.

31. Alexander DH, Lange K. Enhancements to the ADMIXTURE algorithm for individual ancestry estimation. BMC Bioinformatics. 2011 Dec;12(1):246.

32. Goudet J. hierfstat, a package for $r$ to compute and test hierarchical F-statistics. Molecular Ecology Notes. 2005;5(1):184-6.

33. Fischer MC, Foll M, Excoffier L, Heckel G. Enhanced AFLP genome scans detect local adaptation in high-altitude populations of a small rodent (Microtus arvalis). Molecular Ecology. 2011;20(7):1450-62.

34. Whitlock MC, Lotterhos KE. Reliable Detection of Loci Responsible for Local Adaptation: Inference of a Null Model through Trimming the Distribution of F(ST). Am Nat. 2015 Oct;186 Suppl 1:S24-36.

35. Baumann JH, Davies SW, Aichelman HE, Castillo KD. Coral Symbiodinium Community Composition Across the Belize Mesoamerican Barrier Reef System is Influenced by Host Species and Thermal Variability. Microbial Ecology. 2018 May;75(4):903-15.

36. Bushnell B, Rood J, Yao S. BBMap [Internet]. 2018. Available from: sourceforge.net/projects/bbmap/

37. Martin M. Cutadapt removes adapter sequences from high-throughput sequencing reads. EMBnet.journal. 2011 May 2;17(1):10-2.

38. Callahan BJ, McMurdie PJ, Rosen MJ, Han AW, Johnson AJA, Holmes SP. DADA2: High-resolution sample inference from Illumina amplicon data. Nature Methods. 2016 Jul;13(7):581-3.

39. Franklin EC, Stat M, Pochon X, Putnam HM, Gates RD. GeoSymbio: a hybrid, cloudbased web application of global geospatial bioinformatics and ecoinformatics for Symbiodinium-host symbioses: GLOBAL GEOSPATIAL INFORMATICS OF SYMBIODINIUM. Molecular Ecology Resources. 2012 Mar;12(2):369-73. 
40. Frøslev TG, Kjøller R, Bruun HH, Ejrnæs R, Brunbjerg AK, Pietroni C, et al. Algorithm for post-clustering curation of DNA amplicon data yields reliable biodiversity estimates. Nat Commun. 2017 Dec;8(1):1188.

41. McMurdie PJ, Holmes S. phyloseq: An R Package for Reproducible Interactive Analysis and Graphics of Microbiome Census Data. Watson M, editor. PLoS ONE. 2013 Apr 22;8(4):e61217.

42. Green EA, Davies SW, Matz MV, Medina M. Quantifying cryptic Symbiodinium diversity within Orbicella faveolata and Orbicella franksi at the Flower Garden Banks, Gulf of Mexico. PeerJ. 2014 May 13;2:e386.

43. Speare L, Davies SW, Balmonte JP, Baumann J, Castillo KD. Patterns of environmental variability influence coral-associated bacterial and algal communities on the Mesoamerican Barrier Reef. Molecular Ecology [Internet]. 2020 [cited 2020 Jul 23]; Available from: http://onlinelibrary.wiley.com/doi/abs/10.1111/mec.15497

44. Quast C, Pruesse E, Yilmaz P, Gerken J, Schweer T, Yarza P, et al. The SILVA ribosomal RNA gene database project: improved data processing and web-based tools. Nucleic Acids Research. 2012 Nov 27;41(D1):D590-6.

45. Lahti L, Shetty S. microbiome R package [Internet]. 2019. Available from: http://microbiome.github.io

46. Cáceres MD, Legendre P. Associations between species and groups of sites: indices and statistical inference. Ecology. 2009 Dec;90(12):3566-74.

47. Benjamini Y, Hochberg Y. Controlling the False Discovery Rate: A Practical and Powerful Approach to Multiple Testing. Journal of the Royal Statistical Society Series B (Methodological). 1995;57(1):289-300.

48. Narayan NR, Weinmaier T, Laserna-Mendieta EJ, Claesson MJ, Shanahan F, Dabbagh K, et al. Piphillin predicts metagenomic composition and dynamics from DADA2-corrected 16S rDNA sequences. BMC Genomics. 2020 Dec;21(1):56.

49. Kanehisa M, Goto S. KEGG: Kyoto Encyclopedia of Genes and Genomes. Nucleic Acids Research. 2000;28:27-30.

50. Love MI, Huber W, Anders S. Moderated estimation of fold change and dispersion for RNA-seq data with DESeq2. Genome Biol. 2014 Dec;15(12):550.

51. Coelho MAG, Lasker HR. Larval Dispersal and Population Connectivity in Anthozoans. In: Goffredo S, Dubinsky Z, editors. The Cnidaria, Past, Present and Future [Internet]. Cham: Springer International Publishing; 2016 [cited 2019 Mar 4]. p. 291-315. Available from: http://link.springer.com/10.1007/978-3-319-31305-4_19

52. Pérez-Portela R, Riesgo A. Population Genomics of Early-Splitting Lineages of Metazoans. In: Oleksiak MF, Rajora OP, editors. Population Genomics: Marine Organisms [Internet]. Cham: Springer International Publishing; 2018 [cited 2020 Jul 17]. p. 103-37. (Population Genomics). Available from: http://link.springer.com/10.1007/13836_2018_13

53. Lukoschek V, Riginos C, van Oppen MJH. Congruent patterns of connectivity can inform management for broadcast spawning corals on the Great Barrier Reef. Molecular Ecology. 2016;25(13):3065-80.

54. Matz MV, Treml EA, Aglyamova GV, Bay LK. Potential and limits for rapid genetic adaptation to warming in a Great Barrier Reef coral. PLOS Genetics. 2018 Apr 19;14(4):e1007220. 
55. van Oppen MJH, Peplow LM, Kininmonth S, Berkelmans R. Historical and contemporary factors shape the population genetic structure of the broadcast spawning coral, Acropora millepora, on the Great Barrier Reef. Molecular Ecology. 2011;20(23):4899-914.

56. Barshis DJ, Ladner JT, Oliver TA, Seneca FO, Traylor-Knowles N, Palumbi SR. Genomic basis for coral resilience to climate change. Proceedings of the National Academy of Sciences. 2013 Jan 22;110(4):1387-92.

57. Davies SW, Marchetti A, Ries JB, Castillo KD. Thermal and pCO2 Stress Elicit Divergent Transcriptomic Responses in a Resilient Coral. Front Mar Sci [Internet]. 2016 [cited 2020 Jul 22];3. Available from: https://www.frontiersin.org/articles/10.3389/fmars.2016.00112/full

58. Torda G, Donelson JM, Aranda M, Barshis DJ, Bay L, Berumen ML, et al. Rapid adaptive responses to climate change in corals. Nature Climate Change. 2017 Sep 1;7(9):627-36.

59. Epstein HE, Smith HA, Cantin NE, Mocellin VJL, Torda G, van Oppen MJH. Temporal Variation in the Microbiome of Acropora Coral Species Does Not Reflect Seasonality. Frontiers in Microbiology [Internet]. 2019 Aug 16 [cited 2020 Jan 15];10. Available from: https://www.frontiersin.org/article/10.3389/fmicb.2019.01775/full

60. Quigley KM, Davies SW, Kenkel CD, Willis BL, Matz MV, Bay LK. Deep-Sequencing Method for Quantifying Background Abundances of Symbiodinium Types: Exploring the Rare Symbiodinium Biosphere in Reef-Building Corals. Medina M, editor. PLoS ONE. 2014 Apr 11;9(4):e94297.

61. Wainwright BJ, Zahn GL, Afiq-Rosli L, Tanzil JTI, Huang D. Host age is not a consistent predictor of microbial diversity in the coral Porites lutea. Scientific Reports. 2020 Sep $1 ; 10(1): 14376$.

62. Ali A, Kriefall NG, Emery LE, Kenkel CD, Matz MV, Davies SW. Recruit symbiosis establishment and Symbiodiniaceae composition influenced by adult corals and reef sediment. Coral Reefs. 2019 Jun 1;38(3):405-15.

63. Howe-Kerr LI, Bachelot B, Wright RM, Kenkel CD, Bay LK, Correa AMS. Symbiont community diversity is more variable in corals that respond poorly to stress. Global Change Biology. 2020;26(4):2220-34.

64. Fabina NS, Putnam HM, Franklin EC, Stat M, Gates RD. Symbiotic specificity, association patterns, and function determine community responses to global changes: defining critical research areas for coral- Symbiodinium symbioses. Glob Change Biol. 2013 Sep;n/a-n/a.

65. Quigley KM, Bay LK, Willis BL. Temperature and Water Quality-Related Patterns in Sediment-Associated Symbiodinium Communities Impact Symbiont Uptake and Fitness of Juveniles in the Genus Acropora. Front Mar Sci [Internet]. 2017 [cited 2020 Jul 21];4. Available from: https://www.frontiersin.org/articles/10.3389/fmars.2017.00401/full

66. Cooper TF, Berkelmans R, Ulstrup KE, Weeks S, Radford B, Jones AM, et al. Environmental Factors Controlling the Distribution of Symbiodinium Harboured by the Coral Acropora millepora on the Great Barrier Reef. PLOS ONE. 2011 Oct 31;6(10):e25536.

67. Garren M, Walsh SM, Caccone A, Knowlton N. Patterns of association between Symbiodinium and members of the Montastraea annularis species complex on spatial scales ranging from within colonies to between geographic regions. Coral Reefs. 2006 Nov $1 ; 25(4): 503-12$. 
68. Silverstein RN, Cunning R, Baker AC. Change in algal symbiont communities after bleaching, not prior heat exposure, increases heat tolerance of reef corals. Global Change Biology. 2015;21(1):236-49.

69. Hume BCC, Smith EG, Ziegler M, Warrington HJM, Burt JA, LaJeunesse TC, et al. SymPortal: A novel analytical framework and platform for coral algal symbiont nextgeneration sequencing ITS2 profiling. Molecular Ecology Resources. 2019;19(4):1063-80.

70. Davies SW, Moreland K, Wham DC, Kanke MR, Matz MV. Cladocopium community divergence in two Acropora coral hosts across multiple spatial scales. bioRxiv. 2020 May $15 ; 575183$.

71. Thornhill DJ, Howells EJ, Wham DC, Steury TD, Santos SR. Population genetics of reef coral endosymbionts (Symbiodinium, Dinophyceae). Molecular Ecology. 2017;26(10):2640-59.

72. Hernandez-Agreda A, Leggat W, Bongaerts P, Ainsworth TD. The Microbial Signature Provides Insight into the Mechanistic Basis of Coral Success across Reef Habitats. mBio. 2016 Sep 7;7(4):e00560-16, /mbio/7/4/e00560-16.atom.

73. Lima LFO, Weissman M, Reed M, Papudeshi B, Alker AT, Morris MM, et al. Modeling of the Coral Microbiome: the Influence of Temperature and Microbial Network. mBio [Internet]. 2020 Apr 28 [cited 2020 Aug 6];11(2). Available from: https://mbio.asm.org/content/11/2/e02691-19

74. Ziegler M, Seneca FO, Yum LK, Palumbi SR, Voolstra CR. Bacterial community dynamics are linked to patterns of coral heat tolerance. Nat Commun. 2017 Apr;8(1):14213.

75. Thurber RV, Willner-Hall D, Rodriguez-Mueller B, Desnues C, Edwards RA, Angly F, et al. Metagenomic analysis of stressed coral holobionts. Environmental Microbiology. 2009 Aug;11(8):2148-63.

76. Kellogg CA. Microbiomes of stony and soft deep-sea corals share rare core bacteria. Microbiome. 2019 Jun 10;7(1):90.

77. Barott KL, Rodriguez-Brito B, Janouškovec J, Marhaver KL, Smith JE, Keeling P, et al. Microbial diversity associated with four functional groups of benthic reef algae and the reef-building coral Montastraea annularis: Microbial diversity on benthic algae and corals. Environmental Microbiology. 2011 May;13(5):1192-204.

78. Pratte Z, Richardson L. Microbiome dynamics of two differentially resilient corals. Dis Aquat Org. 2018 Nov 20;131(3):213-26.

79. Chapman JA, Kirkness EF, Simakov O, Hampson SE, Mitros T, Weinmaier T, et al. The dynamic genome of Hydra. Nature. 2010 Mar;464(7288):592-6.

80. Franzenburg S, Fraune S, Altrock PM, Künzel S, Baines JF, Traulsen A, et al. Bacterial colonization of Hydra hatchlings follows a robust temporal pattern. ISME J. 2013 Apr;7(4):781-90.

81. Neave MJ, Apprill A, Ferrier-Pagès C, Voolstra CR. Diversity and function of prevalent symbiotic marine bacteria in the genus Endozoicomonas. Appl Microbiol Biotechnol. 2016;100(19):8315-24.

82. Morrow KM, Bourne DG, Humphrey C, Botté ES, Laffy P, Zaneveld J, et al. Natural volcanic $\mathrm{CO} 2$ seeps reveal future trajectories for host-microbial associations in corals and sponges. ISME J. 2015 Mar 17;9(4):894-908.

83. Webster NS, Negri AP, Botté ES, Laffy PW, Flores F, Noonan S, et al. Host-associated coral reef microbes respond to the cumulative pressures of ocean warming and ocean 
acidification. Sci Rep [Internet]. 2016 Jan 13 [cited 2020 Aug 11];6. Available from: https://www.ncbi.nlm.nih.gov/pmc/articles/PMC4725835/ core microbiome identifies rare bacterial taxa as ubiquitous endosymbionts. ISME J. 2015 
FIGURES

628 Figure 1. (a) Map of French Polynesia study sites: Mo’orea NW (MNW), Mo'orea SE (MSE),

629 and Tahiti NW (TNW) (paired back reef and fore reef zones). (b) Daily temperature $\left({ }^{\circ} \mathrm{C}\right)$ ranges

630 at MNW back reef (B) and fore reef (F) from June 2013 - 2014, which overlaps with sampling

631 period (07-08/2013). Data from Service D’Observation CORAIL. (c) Density plots of Acropora

632 hyacinthus colony surface area $\left(\mathrm{cm}^{2}\right.$; Yeo-Johnson-transformed) sampled from fore reef and back

633 reef zones in MNW and TNW (no photographic data available for MSE) with representative

634 photographs from back reef (left) and fore reef (right).

635

(a)

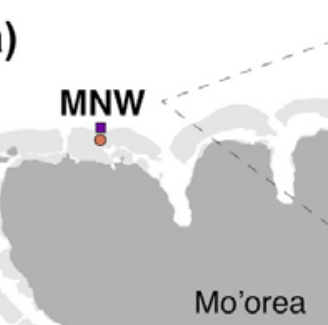

(b)
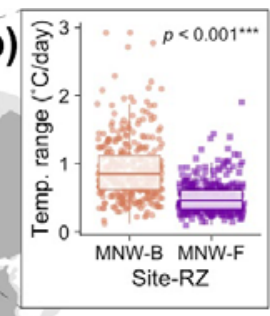

637

638
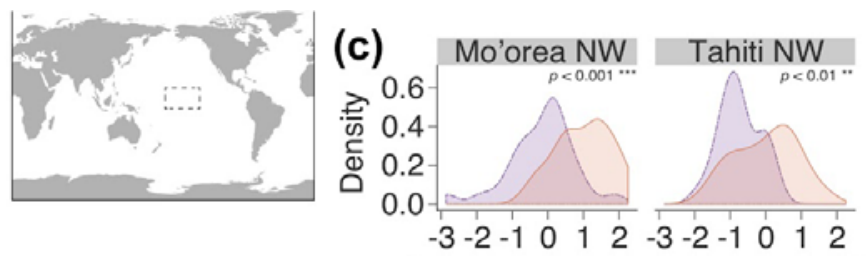

Colony area (Yeo-Johnson transf.)

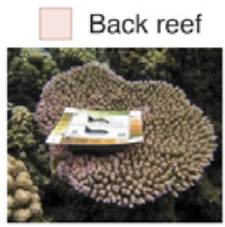

Fore reef

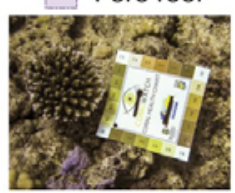


640 Figure 2. (a) Symbiodiniaceae communities in Acropora hyacinthus across reef zones (BR: back

641 reef, FR: fore reef) using multivariate ordination plots (PCoA) based on Bray-Curtis

642 dissimilarity. $P$-values indicate a significant comparison of multivariate location, while $p_{\text {disp }}-$

643 values indicate a significant comparison of dispersion. (b-c) Inverse of Simpson's diversity

644 compared across reef zones, (b) all sites combined and (c) within sites (MNW: Mo'orea NW,

645 MSE: Mo’orea SE, TNW: Tahiti NW.)

646

(a) Mo'orea NW

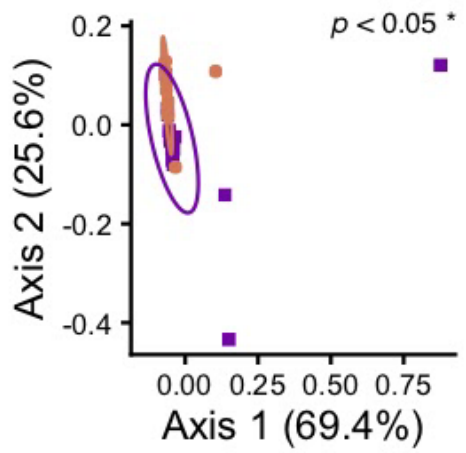

Mo'orea SE

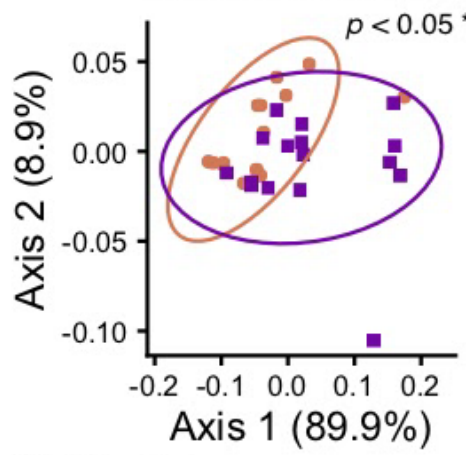

Tahiti NW

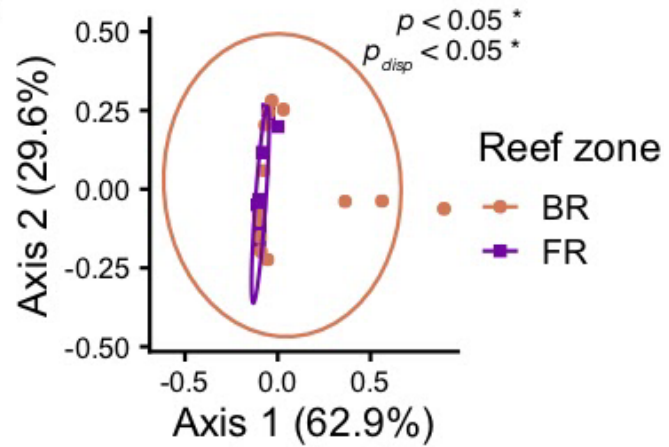

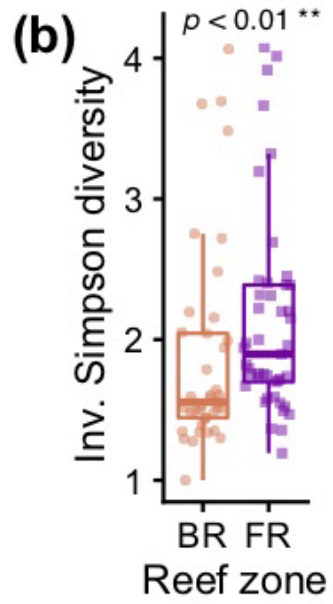

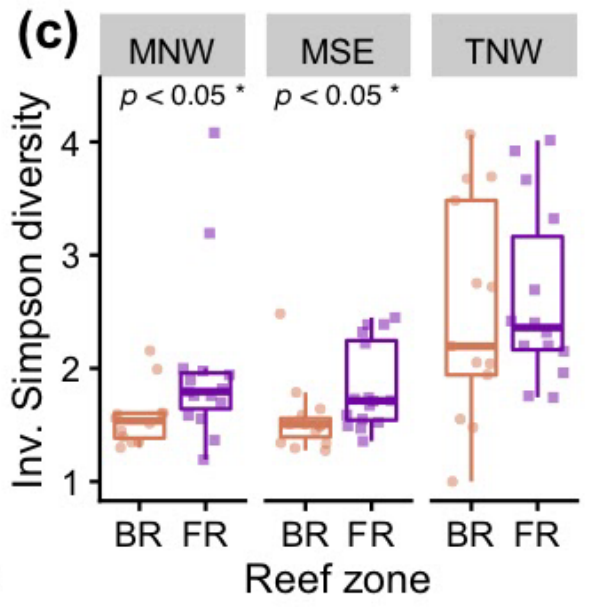

648 
650 Figure 3. Acropora hyacinthus bacterial communities across reef zones (BR: back reef, FR: fore

651 reef) in (a) Mo'orea NW (b) Mo'orea SE and (c) Tahiti NW using multivariate ordination plots

652 (PCoA) based on Bray-Curtis dissimilarity. (d) Average relative abundances, scaled with circle

653 size, of indicator bacterial genera. Genera that appeared as indicators of both back reef and fore

654 reef are marked by asterisks $(*) .(B R:$ back reef, FR: fore reef.)

(a) Mo'orea NW
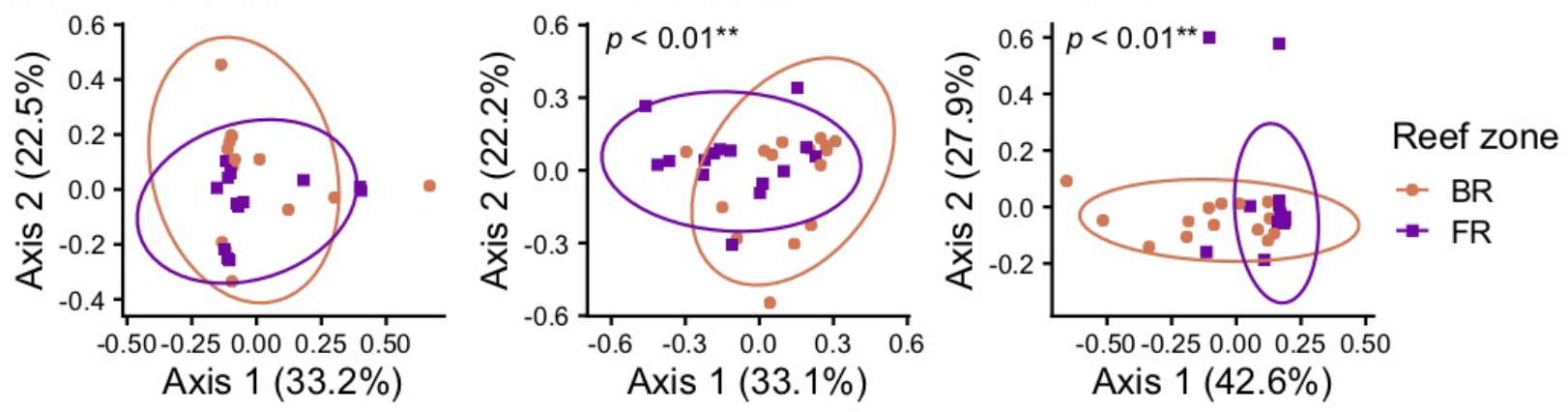

(b)

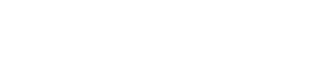

\section{Mo'orea SE

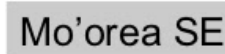

\section{Tahiti NW}

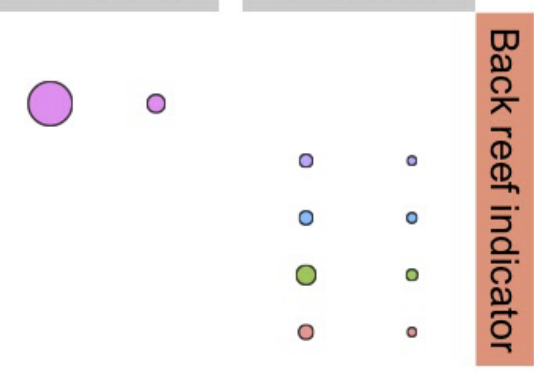

ֻ) Family_Simkaniaceae *Phreatobacter *Lacibacter Enhydrobacter Acidovorax *Phreatobacter *Lacibacter Rubrobacter Curvibacter Endozoicomonas Order_Obscuribacterales Kingdom_Bacteria

\section{BR FR}

Abundance

- 0.000

0.025

0.050

0.075

0.100

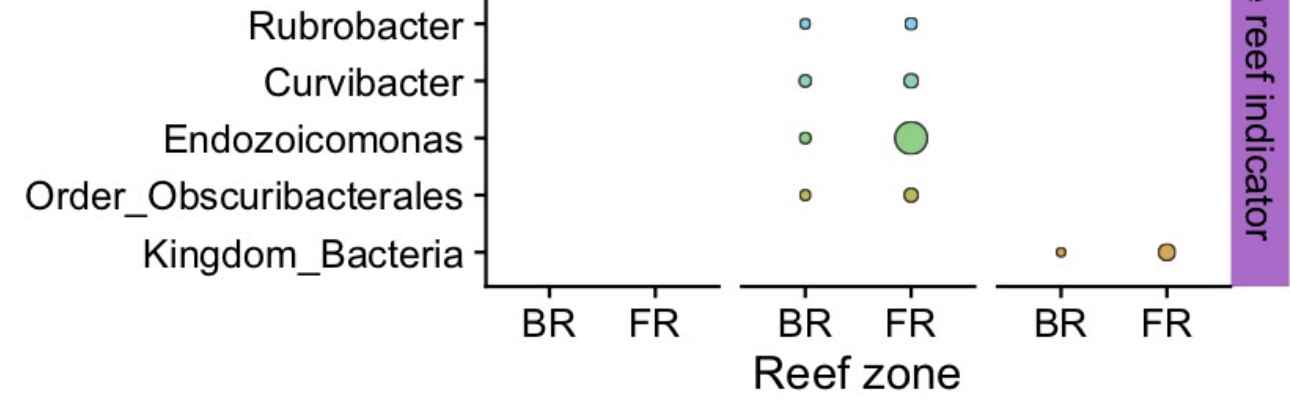


658 Figure 4. Metagenomic profiles inferred from 16S data. (a) Numbers of functional orthologs

659 differentially enriched across reef zones (upper number is back reef, lower number is fore reef)

660 per site. Italicized letters above boxes refer to corresponding panel heat maps. (b) Differentially

661 abundant orthologs shared by Mo'orea NW and Mo'orea SE across the back reef (upper heat

662 map) and fore reef (lower heat map). (c) Differentially abundant orthologs shared by Mo'orea

663 NW and Tahiti NW across the back reef. (BR: back reef, FR: fore reef.)

(a)

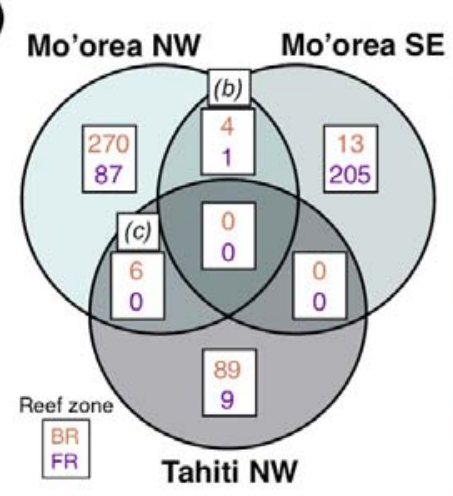

(c)

\section{(b)} Z-score
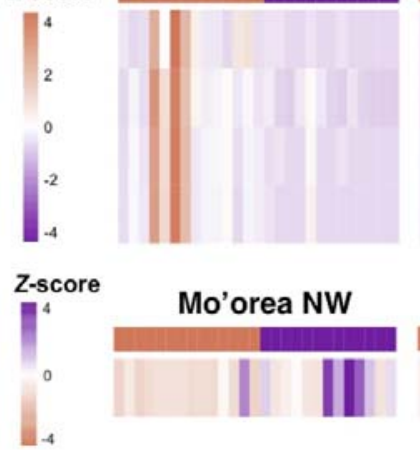

Mo'orea NW
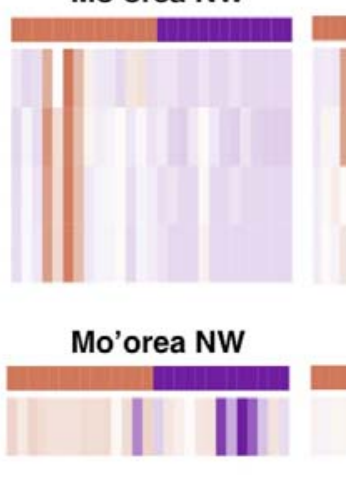

Mo'orea SE

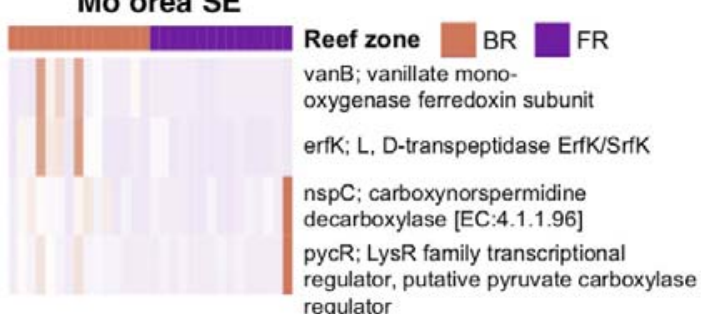

Mo'orea SE

\section{Tahiti NW}

Reef zone $\square$ BR $\square$ FR

ylii; aldose sugar dehydrogenase [EC:1.1.5.-]

aac3-I; aminoglycoside 3-N-acetyltransferase I [EC:2.3.1.60]

tetR; TetR/AcrR family transcriptional regulator, tetracycline repressor protein

aadA; streptomycin 3"-adenylyltransferase [EC:2.7.7.47]

sul1; dihydropteroate synthase type 1 [EC:2.5.1.15]

ebr, qacEdelta1; small multidrug resistance pump 